\title{
MT1JP inhibits glioma progression via negative regulation of $\mathrm{miR}-24$
}

\author{
JINMING CHEN* ${ }^{*}$, JIANYUN LOU* ${ }^{*}$ SHAOCHUN YANG* ${ }^{*}$ JUN LOU, \\ WEI LIAO, RENXIANG ZHOU, CHUANZHEN QIU and GUANFU DING
}

Department of Neurosurgery, The First Affiliated Hospital of Gannan Medical University, Ganzhou, Jiangxi 341000, P.R. China

Received September 28, 2018; Accepted June 12, 2019

DOI: $10.3892 / \mathrm{ol} .2019 .11085$

\begin{abstract}
Long noncoding RNAs have been reported to be dysregulated and have pivotal roles in various human malignancies, including glioma. Previous studies revealed that metallothionein 1J (MT1JP) has important regulatory functions in the development of gastric cancer. However, the biological role and potential mechanism of MT1JP in glioma remain unknown. The present study suggested that MT1JP expression was significantly downregulated in glioma tissues and glioma cell lines, and the decreased expression of MT1JP was associated with glioma progression and poor survival of patients with glioma. Additionally, overexpression of MT1JP significantly inhibited the proliferation and invasion of glioma cells. Furthermore, it was revealed that MT1JP interacted with microRNA-24 (miR-24), which has previously been reported as an oncogene in glioma, negatively regulating its expression level. Rescue experiments revealed that the tumor suppressive functions of MT1JP may be mediated by the negative regulation of miR-24. Collectively, the data suggested that MT1JP inhibited the progression of glioma by negatively regulating miR-24 and may serve as a novel diagnostic biomarker and therapeutic target for glioma.
\end{abstract}

\section{Introduction}

Currently, glioma is the most common and aggressive primary brain tumor in the central nervous system of adults worldwide (1). The yearly incidence of glioma is $\sim 5$ cases per 100,000 individuals and it is also has one of the highest mortality rates worldwide (2-4). Glioma remains a major public health problem in China and worldwide. Therefore,

Correspondence to: Dr Guanfu Ding, Department of Neurosurgery, The First Affiliated Hospital of Gannan Medical University, 23 Youth Road, Ganzhou, Jiangxi 341000, P.R. China

E-mail: cancersci@sina.com

*Contributed equally

Key words: metallothionein 1J, pseudogene, glioma, proliferation, invasion, microRNA-24 it is important to elucidate its underlying mechanisms, and identify novel diagnostic biomarkers and therapeutic targets for glioma.

Long non-coding RNA (lncRNA) is a class of ncRNAs of $>200$ nucleotides that are poorly conserved in different species (5). IncRNAs were once considered to be transcriptional 'noise' without biological functions, as the RNAs have no evident protein-coding capacity (6). However, increasing evidence has demonstrated that lncRNAs have pivotal roles in the initiation and progression of human malignancies, including glioma, involving transcriptional and post-transcriptional regulation $(7,8)$.

lncRNA metallothionein 1J, pseudogene (MT1JP) is located on chromosome 16 in a cluster consisting of several homologous protein-coding genes. Liu et al (9) reported the tumor suppressive function of MJ1JP and revealed that MT1JP exerts its roles by regulating the expression of $\mathrm{p} 53$ and, therefore, regulating the activity of the p53 signalling pathway. Subsequently, Xu et al (10) reported that MT1JP is downregulated in gastric cancer tissues and cell lines. Additionally, low expression of MT1JP was significantly associated with advanced Tumor, Necrosis and Metastasis stage and lymphatic metastasis (10). Furthermore, MT1JP overexpression suppresses gastric cancer cell proliferation, migration and invasion, and promoted apoptosis in vitro and in vivo via an MT1JP/microRNA (miRNA)-214-3p/runt related transcription factor 3 axis (10); however, the biological functions and potential mechanisms of MT1JP in glioma remain unclear.

In the present study, the expression of MT1JP was investigated in glioma tissues and cell lines, and its biological functions and underlying mechanisms were explored. The present results suggested that MT1JP was downregulated in glioma and its overexpression inhibited glioma cell proliferation, migration and invasion by negatively regulating miRNA-24 (miR-24). The present data suggested that MT1JP may have an important tumor-suppressing role in glioma and may serve as a novel diagnostic biomarker and therapeutic target for glioma.

\section{Materials and methods}

Human tissue samples. Between January 2006 and December 2011, 40 pairs of glioma tissues and paired adjacent normal tissues were collected from patients (23 males, 
17 females; range, 37-68 years) undergoing resection surgery at the Department of Neurosurgery, The First Affiliated Hospital of Gannan Medical University (Ganzhou, China). The inclusion criteria were that patients were aged $>18$ years, and had a pathological diagnosis of glioma in the brain based on the WHO classification (11). The exclusion criteria were that the patient had another type of tumor, or an infection or non-surgical removal of the tumor. The adjacent normal brain tissues were obtained $2-3 \mathrm{~cm}$ away from the glioma during surgery. Histological examination was performed in order to verify the normal brain tissues and glioma tissues. No patients received preoperative anti-cancer treatment, including chemotherapy or radiation, prior to the collection of specimens. All specimens from resection surgery were frozen and stored in liquid nitrogen $\left(-196^{\circ} \mathrm{C}\right)$ until required. The present study was performed with the approval of the Ethic and Research Committees of the First Affiliated Hospital of Gannan Medical University. Informed consent was obtained from all participants.

Cell culture. Human glioma cell lines (SHG-44 and U251), glioblastoma of unknown origin (U87; cat. no. HTB14) and human astroglia cells (HA) were used in the present study. All cell lines were purchased from American Type Culture Collection and cultured in DMEM (Gibco; Thermo Fisher Scientific, Inc.) supplemented with $10 \%$ FBS, $50 \mathrm{U} / \mathrm{ml}$ penicillin and $0.1 \mathrm{mg} / \mathrm{ml}$ streptomycin (Biowest). All the cell cultures were incubated at $37^{\circ} \mathrm{C}$ in $5 \% \mathrm{CO}_{2}$.

$R N A$ isolation and reverse transcription-quantitative $P C R$ $(R T-q P C R)$. Total RNA was extracted from glioma tissues and glioma cell lines (80-90\% confluence) using TRIzol ${ }^{\circledR}$ reagent (Invitrogen; Thermo Fisher Scientific, Inc.) according to the manufacturer's instructions. Following extraction, the RNA and miRNA samples were reverse transcribed into cDNA using a RevertAid RT kit (Thermo Fisher Scientific, Inc.) or miRNA RT kit (Tiangen Biotech Co., Ltd.), respectively. The reverse transcription reaction condition was as follows: $37^{\circ} \mathrm{C}$ for $15 \mathrm{~min}, 85^{\circ} \mathrm{C}$ for $5 \mathrm{sec}$ and $4^{\circ} \mathrm{C}$ for $2 \mathrm{~min}$. The cDNA templates were amplified by qPCR using SYBR Green Mix (Thermo Fisher Scientific, Inc.). The following thermocycling conditions were used for the qPCR: Initial denaturation at $95^{\circ} \mathrm{C}$ for $55 \mathrm{sec}, 40$ cycles of $95^{\circ} \mathrm{C}$ for $22 \mathrm{sec}$ and $60^{\circ} \mathrm{C}$ for $44 \mathrm{sec}$. mRNA levels were quantified using the $2^{-\Delta \Delta \mathrm{Cq}}$ method (12). GAPDH was used as an internal control for IncRNAs and miRNA samples were normalized to U6 expression. The following primers were used for the qPCR: MT1JP, forward: 5'-TACCGAGCTCGGATCCTTGCGGTC TCTCCATTTATCG-3', reverse: 5'-TACCGAGCTCGGATC CTTGCGGTCTCTCCATTTATCG-3'; GAPDH, forward: 5'-GCACCGTCAAGGCTGAGAAC-3', reverse: 5'-TGGTGA AGACGCCAGTGGA-3'. U6 forward, 5'-CTCGCTTCGGCA GCACA3' and reverse, 5'-AACGCTTCACGAATTTGCGT-3'. The aforementioned primers were designed and synthesized by Shangai GenePharma Co., Ltd. (Shanghai, China).

Constructs, synthesized oligos and transfection. The MT1JP overexpression plasmid and miR-24 mimic were purchased from Shanghai GenePharma Co., Ltd. The plasmids and mimics were both introduced into cells at a final concentration of $50 \mathrm{nM}$. The sequence was amplified and inserted into the pcDNA3.1 (+) vector at the BamH1 sites. The pcDNA3.1 (+) empty vector (Shanghai GenePharma Co., Ltd) was used as a negative control for MT1JP overexpression. Lipofectamine ${ }^{\circledR} 3000$ (Thermo Fisher Scientific, Inc.) was used for transfection according to the manufacturer's instructions. At $48 \mathrm{~h}$ after transfection, cells were used for the subsequent experiments. The sequence of the negative control mimic was as follows: 5'-UUCUCCGAACGUGUC ACGUTT-3'.

Cell proliferation assay. The cell proliferation assay was performed using a Cell Counting Kit-8 (CCK-8; Dojindo Molecular Technologies, Inc.) according to the manufacturer's protocol. Cells were placed into the 96 well plates at a density of $\sim 3,000$ cells/well, and examined every $24 \mathrm{~h}$. The absorbance was recorded at $450 \mathrm{~nm}$ and analyzed following the instructions provided.

Cell invasion assay. Cell invasion was assessed using a Matrigel assay. Cells $\left(\sim 1 \times 10^{5}\right)$ were seeded into the upper chambers of a Transwell plate (Corning, Inc.) with Matrigel (Corning, Inc.) in $300 \mu \mathrm{l}$ serum-free DMEM, and the lower chambers were filled with $700 \mu \mathrm{l}$ DMEM containing $10 \%$ FBS. After $6 \mathrm{~h}$ of incubation at $37^{\circ} \mathrm{C}$, the filters were treated with $4 \%$ paraformaldehyde for $15 \mathrm{~min}$ at room temperature and crystal violet for $10 \mathrm{~min}$ at room temperature. Finally, the number of cells that invaded through the membrane was counted and calculated in $\geq 5$ randomly selected fields of view under an Olympus CKX31 inverted light microscope (magnification, x400; Olympus Corporation). Image J (version 1.40; National Institutes for Health) software was used to quantify cell number.

Dual-luciferase reporter assay. The potential binding sites were mutated by the QuikChang site-directed mutagenesis kit (Agilent Technologies, Inc.). The wild type MT1JP (WT) and mutant MT1JP (MUT) sequence containing the seeding site of miR-24 were established and incorporated into the Firefly luciferase expressing psiCHECK-2 vector (Promega Corporation). All the cloned sequences were validated by DNA sequencing. U87 cells were seeded into a 24 -well plate for $24 \mathrm{~h}$ before transfection. Cells were transfected with $0.4 \mu \mathrm{g}$ pMiR-report-MT1JP-WT or pMiR-report-MT1JP-MUT plasmid, and $20 \mu \mathrm{M}$ miR-24 mimic or control scrambled oligo (Shanghai GenePharma Co., Ltd.; sequence: 5'-UUCUCCGAACGUGUCACGUTT-3'), together with $0.02 \mu \mathrm{g}$ Renilla luciferase vector pRL-TK (Promega Corporation) using Lipofectamine ${ }^{\circledR} 2000$ (Invitrogen; Thermo Fisher Scientific, Inc.). After 48 h, the luciferase activities were detected consecutively using the Dual-Luciferase ${ }^{\circledR}$ Reporter assay system (Promega Corporation) following the manufacturer's instructions.

Statistical analysis. All results are presented as the mean \pm SD and analysed using GraphPad Prism 5 (GraphPad Software, Inc.) from $\geq 3$ independent experiments. The $\chi^{2}$ test was performed to examine the associations between MT1JP level and clinicopathological features. The Kaplan-Meier method was used to calculate the survival curve, and log-rank test was used to determine statistical significance. The differences 
A

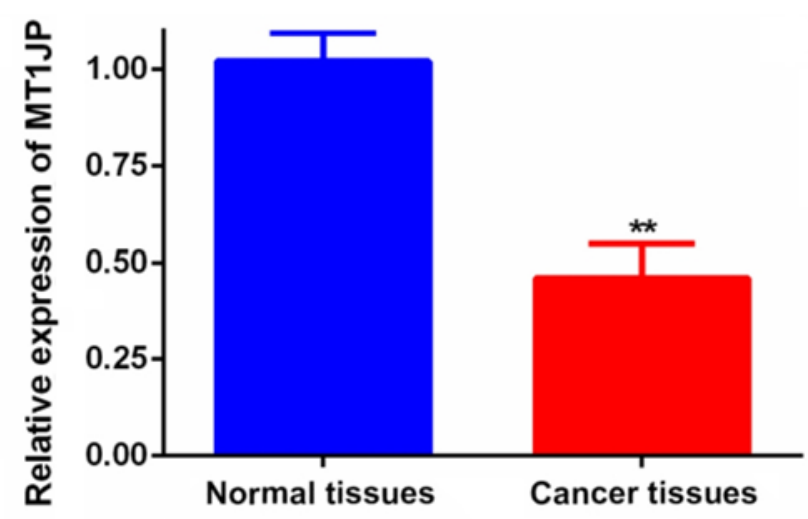

C

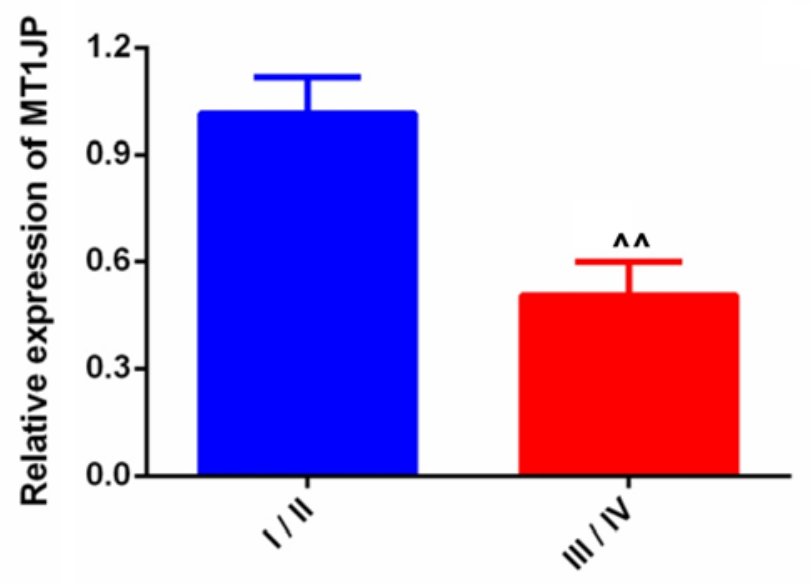

B
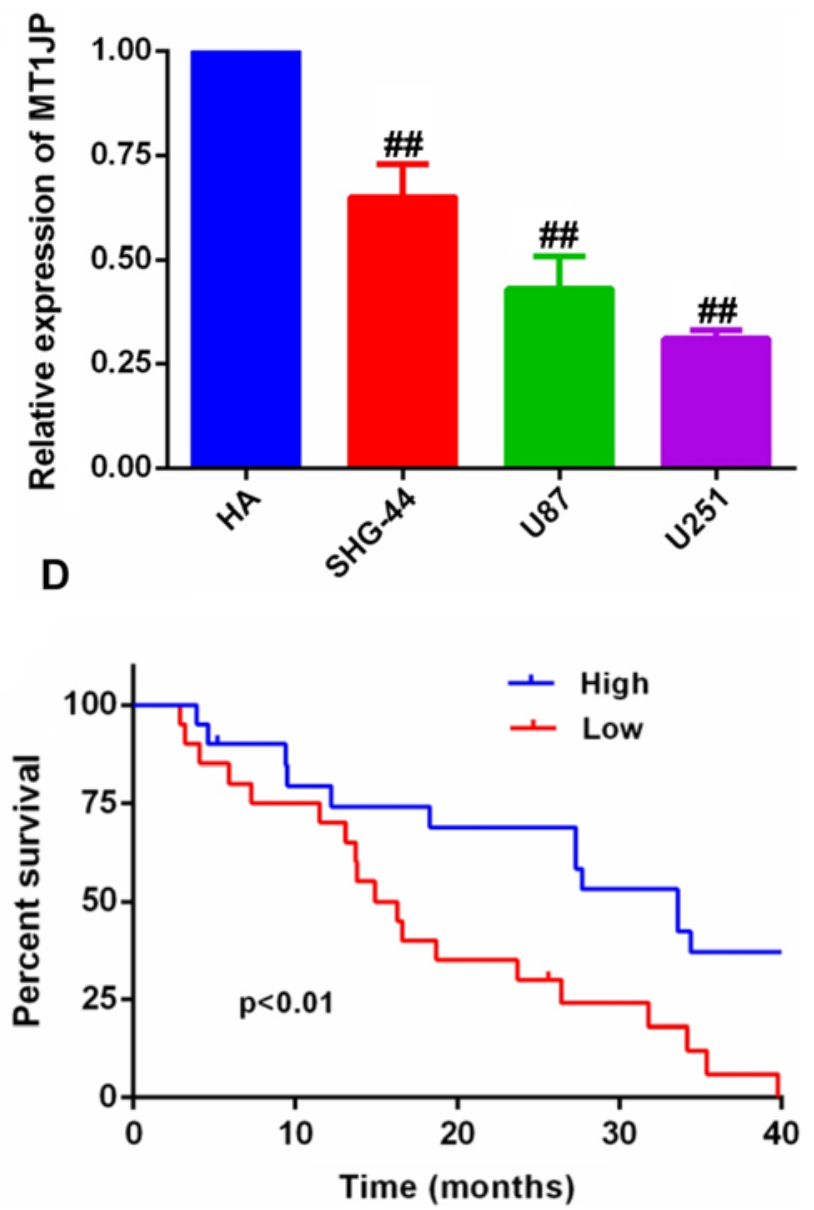

Figure 1. MT1JP expression is decreased in glioma tissues and cell lines. (A) Relative mRNA expression of MT1JP in 40 pairs of glioma tissue and adjacent non-tumor tissues by reverse transcription-quantitative PCR analysis. (B) Downregulation of MT1JP in glioma cells compared with that in HA cells. (C) Expression of MT1JP was lower in the higher WHO graded tumors compared with that in the lower WHO graded samples. (D) Kaplan-Meier survival curves for patients with glioma expressing low and high levels of MT1JP. ${ }^{* *} \mathrm{P}<0.01$ vs. normal tissue; ${ }^{\# \#} \mathrm{P}<0.01$ vs. HA cells; ${ }^{\wedge} \mathrm{P}<0.01$ vs. I/II stages. MT1JP, metallothionein 1J; WHO, World Health Organisation.

among groups were analyzed using one-way ANOVA followed by Tukey's post-hoc test. Expression correlations were analyzed using Pearson's correlation coefficient. $\mathrm{P}<0.05$ was considered to indicate a statistically significant difference.

\section{Results}

MT1JP is downregulated in glioma. MT1JP expression was determined in glioma tissues and cell lines. The results indicated that MT1JP expression was downregulated in glioma tissues (Fig. 1A) and cell lines (Fig. 1B). Furthermore, the expression of MT1JP was lower in samples presenting higher World Health Organisation (WHO) (11) tumor grades compared with lower WHO grades (Fig. 1C). Additionally, Kaplan-Meier survival analysis (Fig. 1D) suggested that the overall survival time of patients with glioma was improved in the higher MT1JP expression group compared with that in the lower expression group. The mean value was used to determine high and low expression of MT1JP.

Overexpression of MT1JP suppresses glioma cell proliferation and invasion. RT-qPCR revealed that the expression of MT1JP was significantly upregulated in U87 (Fig. 2A) and U251 cells (Fig. 2B) transfected with pcDNA-MT1JP compared with control. The CCK-8 assay suggested that overexpression of MT1JP significantly inhibited glioma cell proliferation (Fig. 2C and D). Additionally, the Matrigel invasion assay indicated that overexpression of MT1JP significantly inhibited glioma cell invasion (Fig. 3).

miR-24 expression in glioma. Overexpression of MT1JP suppressed miR-24 expression in U87 cells (Fig. 4A) and U251 cells (Fig. 4B). Additionally, the expression level of miR-24 in glioma and paired adjacent normal tissues was evaluated by RT-qPCR. The miR-24 expression level was significantly upregulated in glioma tissues compared with that in the control normal tissues (Fig. 5A). Furthermore, the expression of miR-24 was higher in the higher WHO graded samples compared with that in the lower WHO graded samples (Fig. 5B).

Furthermore, miR-24 expression was significantly upregulated in the glioma cell lines SHG-44, U87 and U251 compared with HA cells (Fig. 5C). Notably, miR-24 expression was negatively correlated with MT1JP expression in 

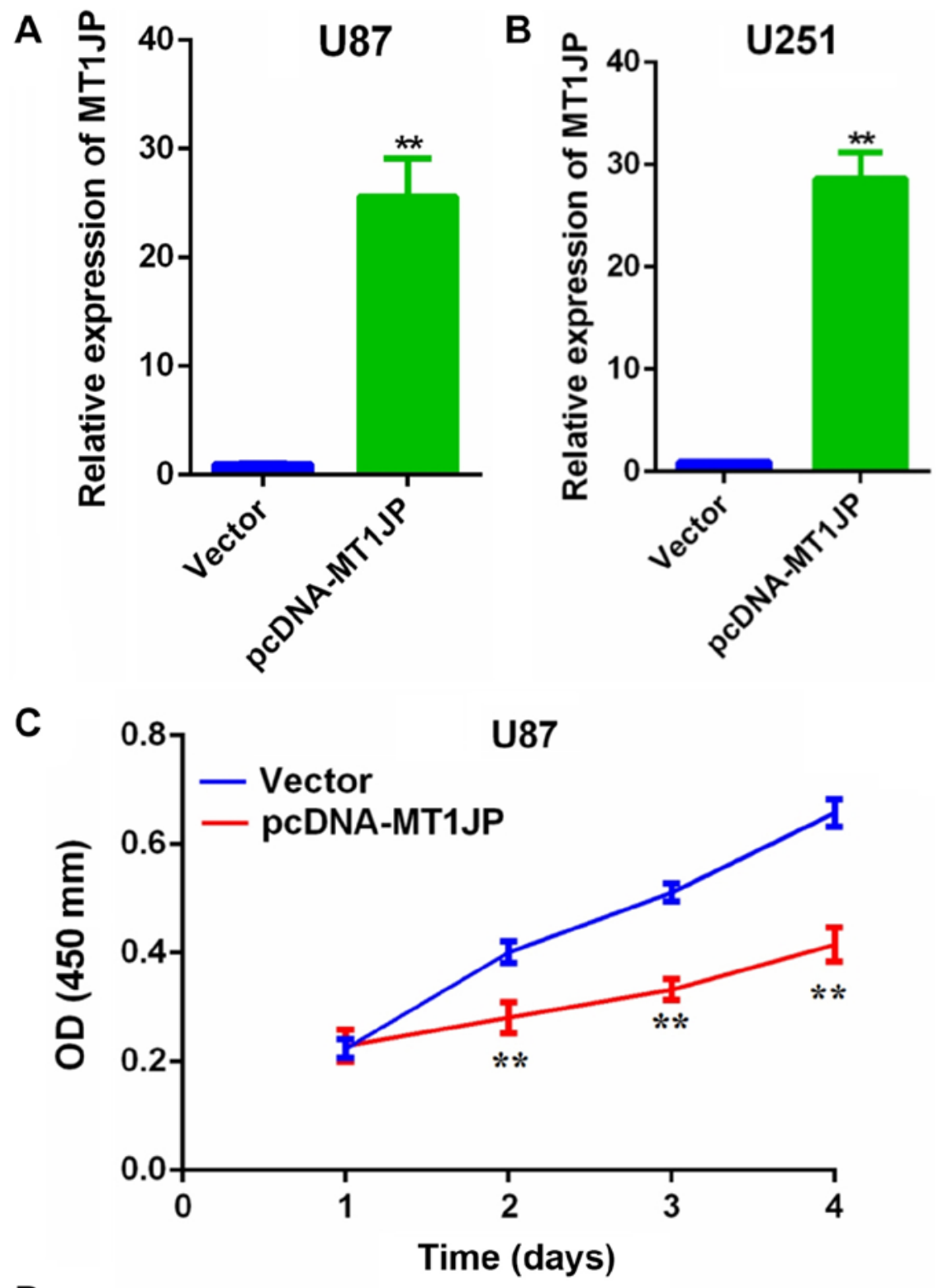

D

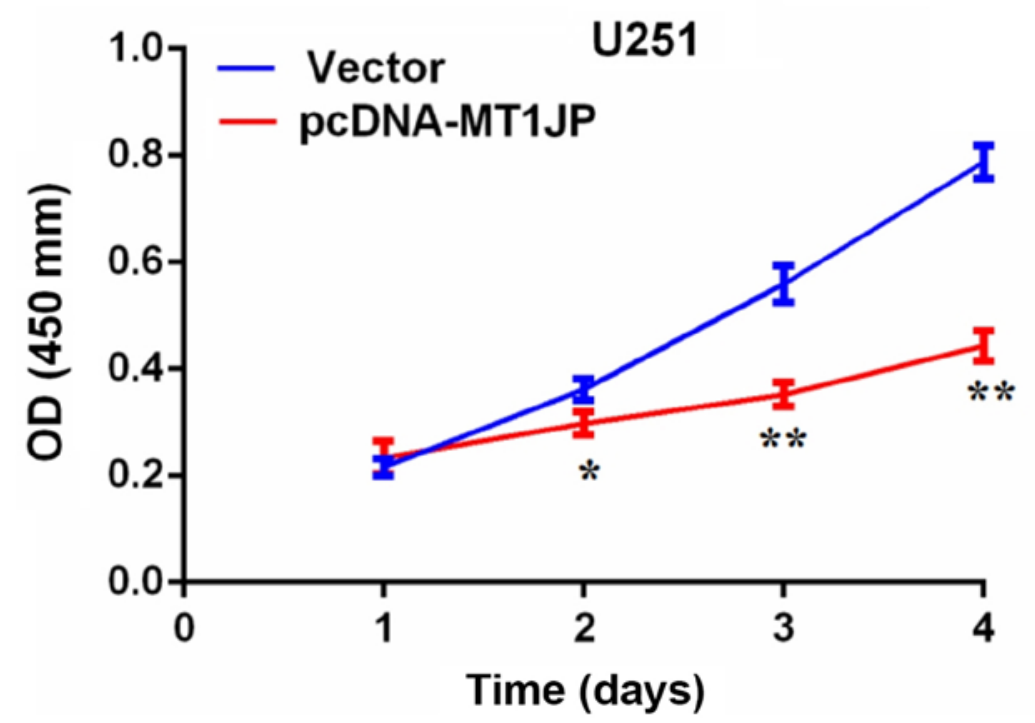

Figure 2. MT1JP overexpression inhibits proliferation of glioma cells. (A) Relative mRNA expression level of MT1JP in U87 cells transfected with pcDNA-MT1JP. (B) Relative expression level of MT1JP in U251 cells transfected with pcDNA-MT1JP. ${ }^{* *} \mathrm{P}<0.01$ vs. empty vector. (C) MT1JP overexpression suppressed U87 cell proliferation. (D) MT1JP overexpression suppressed U251 cell proliferation. "P<0.01 vs. empty vector. MT1JP, metallothionein $1 \mathrm{~J}$; OD, optical density. 

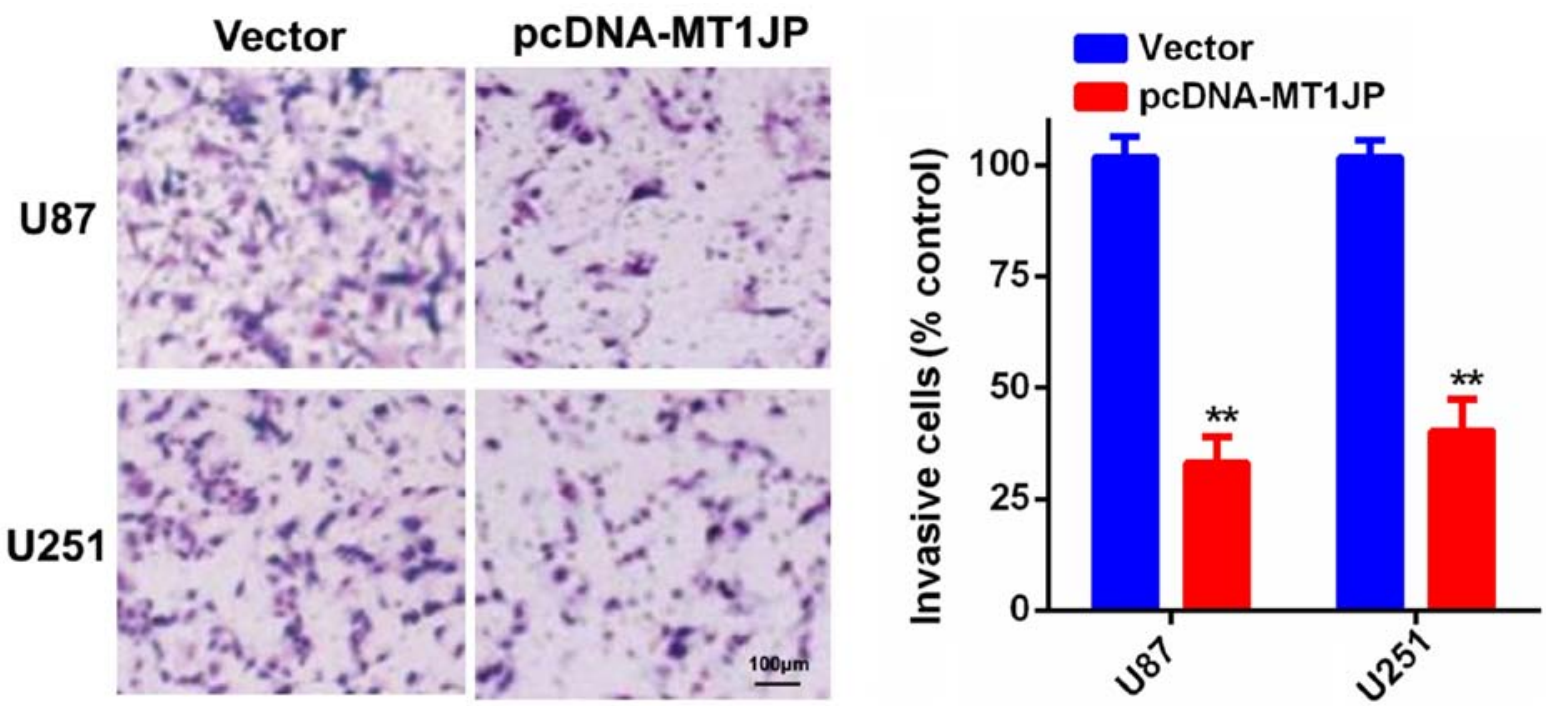

Figure 3. MT1JP overexpression suppresses glioma cells invasion. pcDNA-MT1JP significantly increased MT1JP expression and MT1JP inhibited U87 and U251 cells invasion. ${ }^{* *} \mathrm{P}<0.01$ vs. empty vector. MT1JP, metallothionein $1 \mathrm{~J}$.
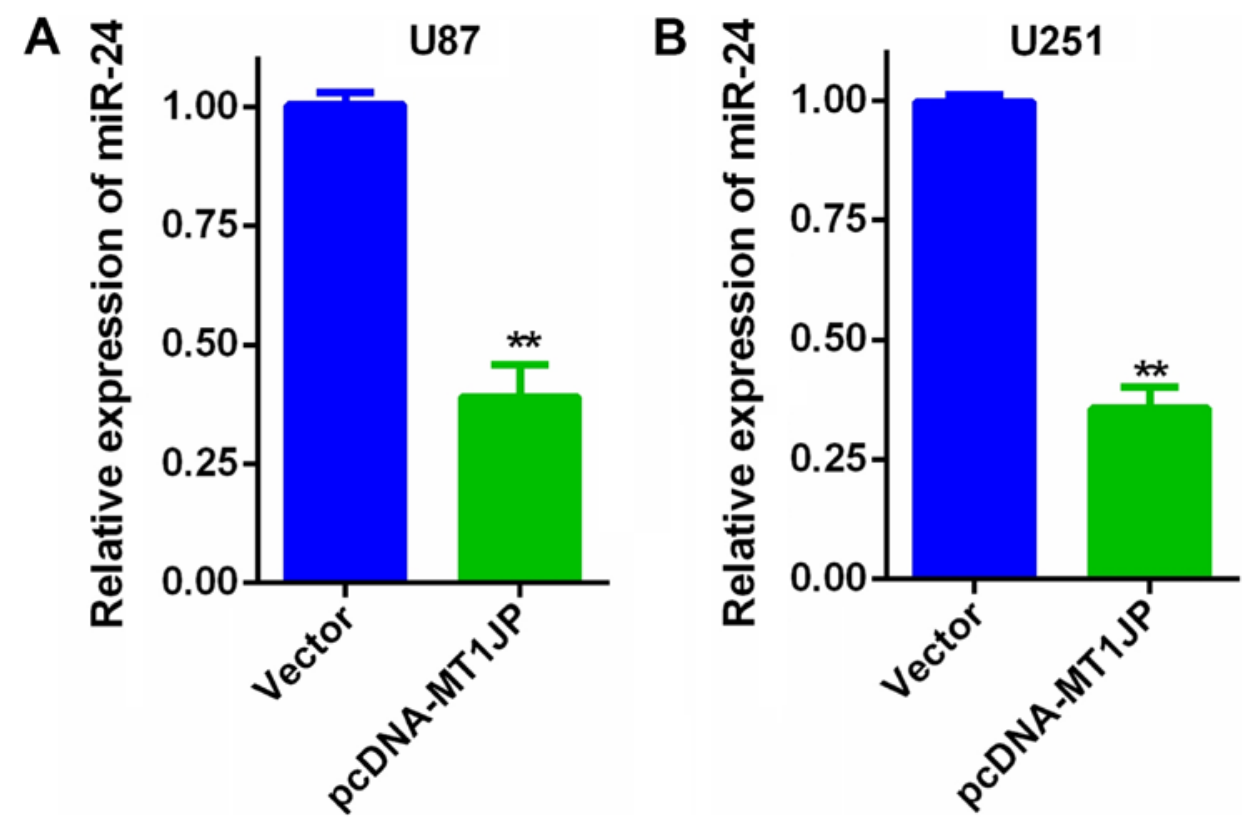

Figure 4. MT1JP overexpression suppresses miR-24 expression in (A) U87 cells and (B) U251 cells. ${ }^{* *} \mathrm{P}<0.01$ vs. empty vector. MT1JP, metallothionein $1 \mathrm{~J}$; miR, microRNA.

glioma tissues (Fig. 5D). Finally, Kaplan-Meier survival analysis suggested that the overall survival time of patients with glioma was significantly improved in patients with lower miR-24 expression compared with that in the higher expression group (Fig. 5E).

MT1JP is a direct target of miR-24. Bioinformatics analysis of the predicted IncRNA-miRNA interactions revealed potential binding sequences between MT1JP and miR-24 (Fig. 6A) (10). Subsequently, a luciferase reporter assay confirmed that miR-24 overexpression significantly decreased the luciferase activity of the MT1JP-WT reporter compared with a negative control oligo, whereas miR-24 did not affect the luciferase activity of the MT1JP-MUT reporter in U87 cells (Fig. 6B). In addition, following transfection with miR-24 mimics, the mRNA expression level of miR-24 in both U87 and U251 cells was significantly increased (Fig. 6C).

Inhibitory effect of MT1JP on glioma cell proliferation and invasion is dependent on miR-24. Rescue experiments were performed to determine whether MT1JP-mediated inhibition of glioma cell proliferation and invasion was miR-24-dependent. miR-NC or miR-24 mimic were co-transfected into U87 cells transfected with pcDNA-MT1JP. These results confirmed that miR-NC or miR-24 mimic was stably transfected into U87 cells that were already transfected with pcDNA-MT1JP (Fig. 7A). CCK-8 and Matrigel 

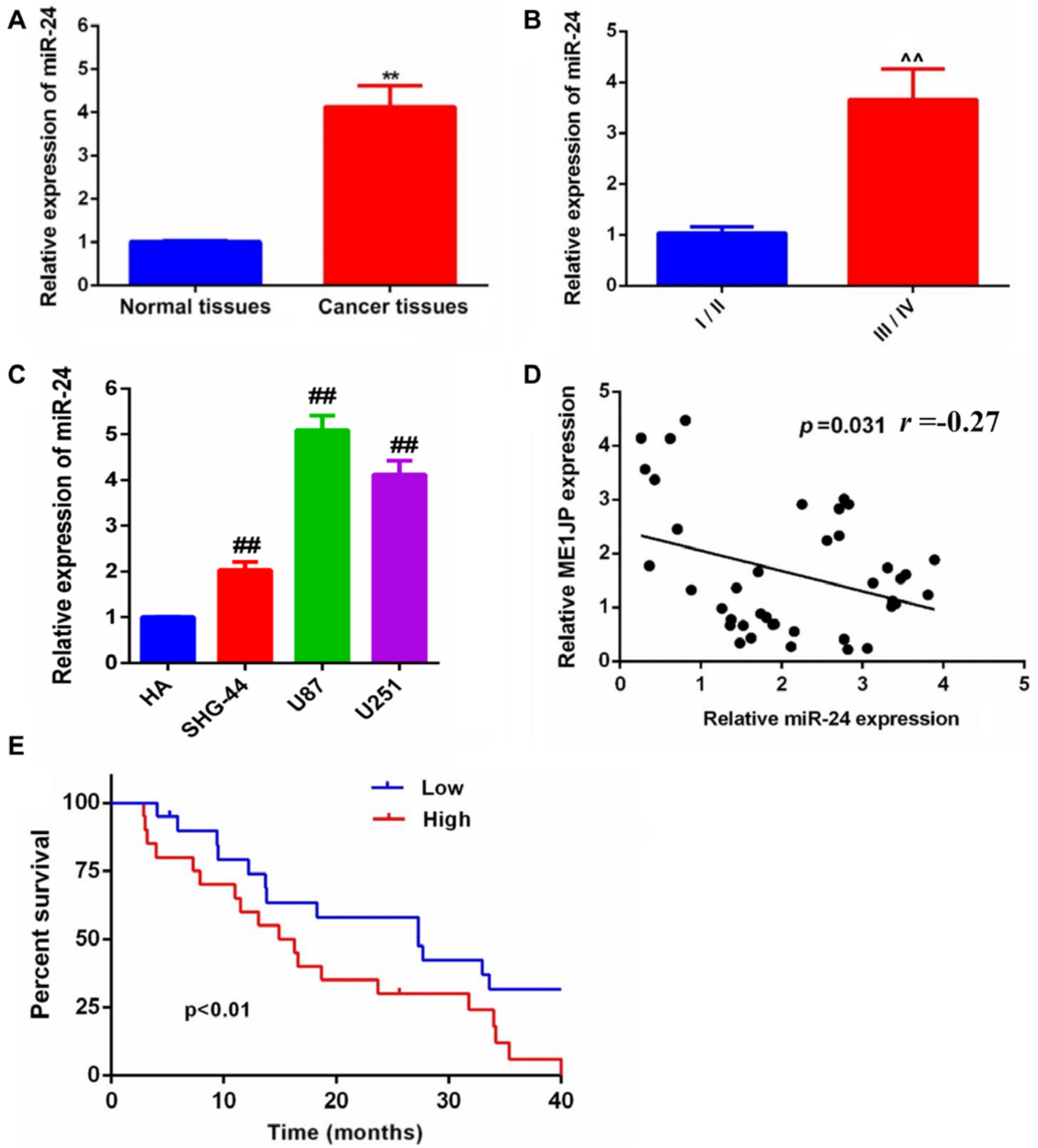

Figure 5. miR-24 is upregulated in glioma tissues and cell lines. (A) Expression of miR-24 in the glioma tissues and normal tissues was measured using RT-qPCR. (B) expression of miR-24 was higher in high WHO graded samples compared with that in lower WHO graded samples. ${ }^{\wedge} \mathrm{P}<0.01 \mathrm{vs}$. I/II stage. (C) mRNA expression of miR-24 in the glioma cell lines and HA cells were measured using RT-qPCR. (D) mRNA expression level of miR-24 was inversely correlated with MT1JP expression level in glioma tissues. (E) Kaplan-Meier survival curves for patients with glioma expressing low and high levels of miR-24. ${ }^{* *} \mathrm{P}<0.01$ vs. normal tissue; ${ }^{\# \#} \mathrm{P}<0.01$ vs. HA cells; ${ }^{\wedge} \mathrm{P}<0.01$ vs. I/II stages. miR, microRNA; RT-qPCR, reverse transcription-quantitative PCR; MT1JP, metallothionein $1 \mathrm{~J}$.

invasion assays suggested that the inhibition of proliferation and invasion of U87 cells that was induced by MT1JP overexpression was partially abolished in the presence of the miR-24-3p mimic (Fig. 7B and C). These data indicated that the tumor suppressive function of MT1JP in glioma cells involved the negative regulation of $\mathrm{miR}-24$.

\section{Discussion}

Glioma cells were reported to carry heterogeneous genetic molecular aberrations and the molecular mechanism of glioma development is highly complex $(13,14)$. An increasing number of studies have revealed that lncRNAs 

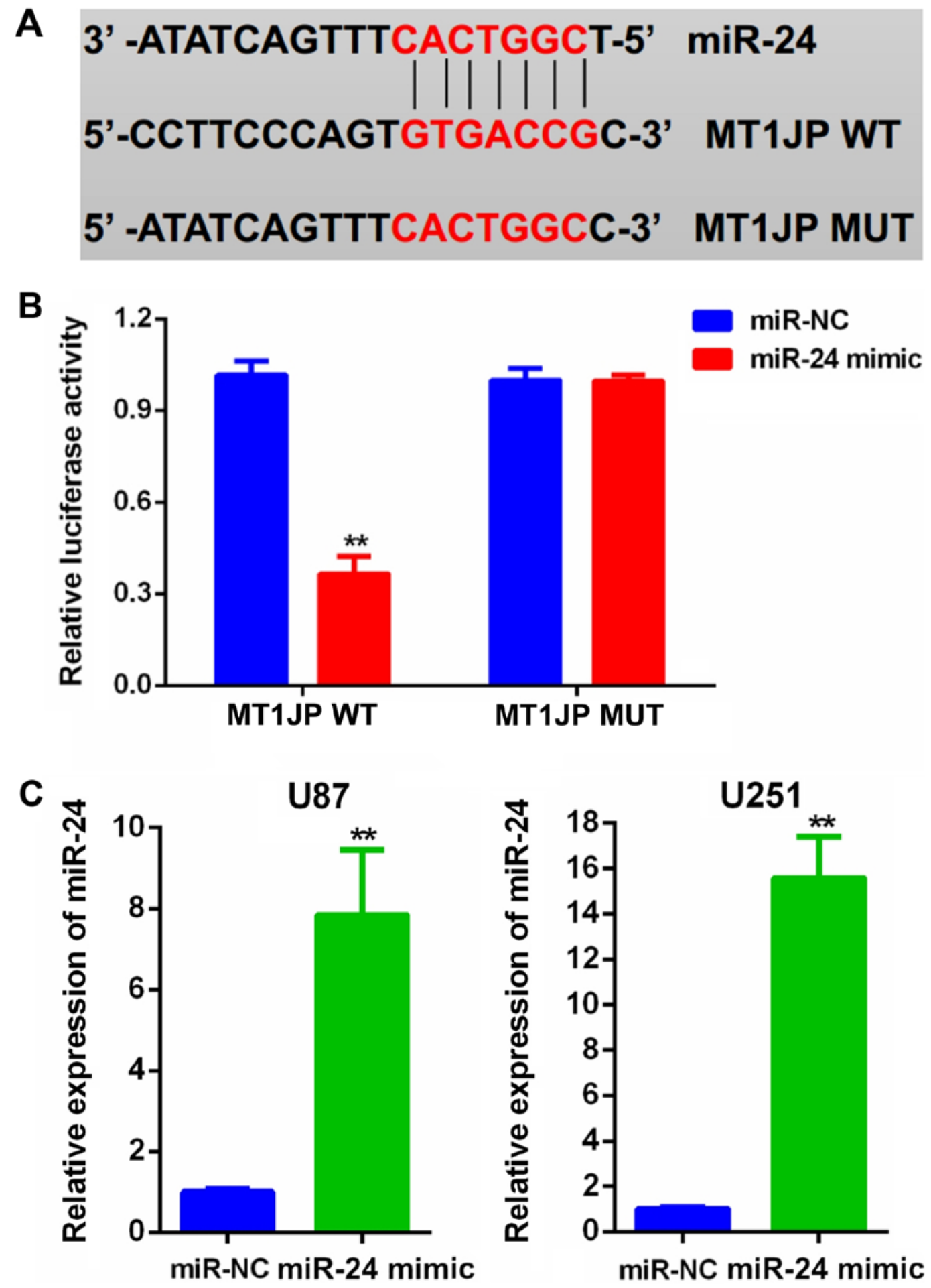

Figure 6. miR-24 is a target of MT1JP. (A) Putative complementary sites between miR-24 and MT1JP. (B) Luciferase activity in U87 cells co-transfected with the miR-24 mimic or miR-NC luciferase reporters with MT1JP WT or MT1JP MUT insert. (C) Relative mRNA expression level of miR-24 in U87 and U251 cells transfected with miR-24 mimic. ${ }^{* *} \mathrm{P}<0.01$ vs. miR-NC. miR, microRNA; MT1JP, metallothionein $1 \mathrm{~J}$; NC, negative control; WT, wild type; MUT, mutant.

have pivotal roles in the development of glioma (15-18). lncRNA small nucleolar RNA host gene 12 promotes glioma progression via an miR-101-3p/forkhead box P1 axis (19). Long intergenic non-coding RNA 152 facilitates glioma cell proliferation and invasion by interacting with miR-16 (20).

The present study demonstrated that MT1JP expression was significantly decreased in glioma tissues and cell lines.
The decreased expression of MT1JP was significantly associated with poor prognosis. Furthermore, overexpression of MT1JP inhibited the proliferation and invasion of glioma cells. These data suggest that MT1JP has pivotal roles in glioma development.

IncRNAs can regulate the development of various human malignancies, including glioma, via several different mechanisms, including transcriptional and post-transcriptional 


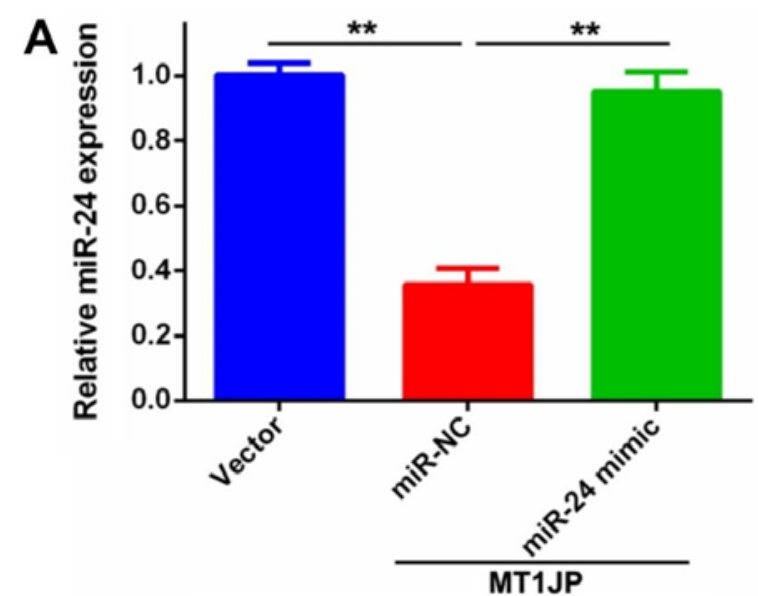

C

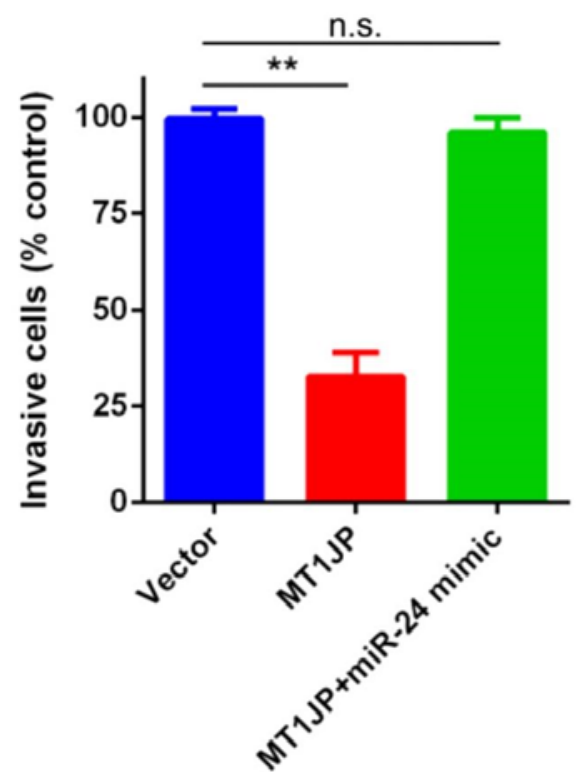

B

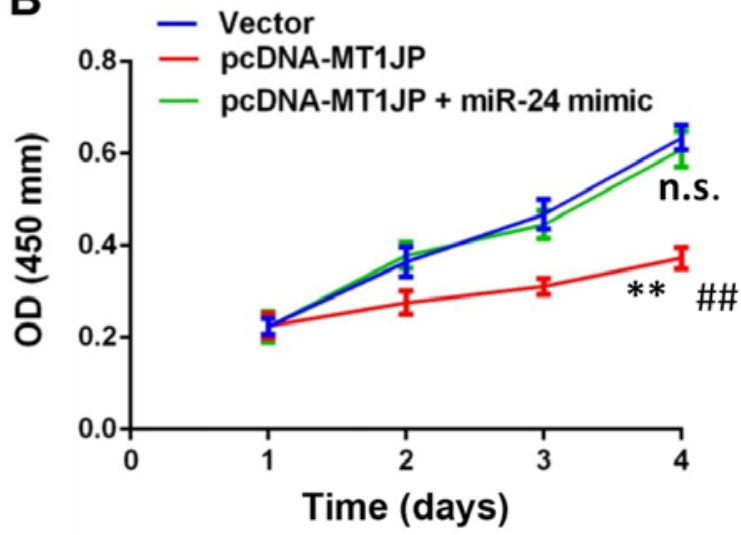

Figure 7. Tumor suppressive function of MT1JP is dependent on miR-24. (A) Reverse transcription-quantitative polymerase chain reaction was used to measure miR-24 expression in U87 cells that were stably co-transfected with pcDNA-MT1JP and miR-24 mimic or miR-NC. ${ }^{* *} \mathrm{P}<0.01$. (B) Cell Counting Kit-8 assays were performed to measure proliferation of U87 cells that were stably transfected with pcDNA-MT1JP and miR-24 mimic or NC. n.s., not siginificant; ${ }^{* *}<<0.01$ vs. vector; ${ }^{\# \#} \mathrm{P}<0.01$ vs. pcDNA-MT1JP+miR-44 mimic. (C) Matrigel assays were performed to measure invasion of U87 cells that were stably transfected with pcDNA-MT1JP and miR-24 mimic or an empty plasmid. ${ }^{* * *} \mathrm{P}<0.01$. MT1JP, metallothionein $1 \mathrm{~J}$; miR, microRNA; NC, negative control; n.s., not significant.

regulations $(21,22)$. Accumulating evidence has revealed that IncRNAs function as a competing endogenous RNA or a molecular sponge to regulate the expression and biological functions of miRNAs $(23,24)$. For example, IncRNA retinal non-coding RNA 3 promotes prostate cancer development via regulation of miR-185 (25). LncRNA X inactive specific transcript sponges miR-34a, facilitating colon cancer development (26). A previous study demonstrated that MT1JP inhibits proliferation, migration and invasion, and promotes apoptosis of gastric cancer cells (10). Bioinformatics analysis suggested that miR-24 was a potential target of MT1JP, and was previously reported as a oncogenic miRNA in glioma (27). Thus, it was hypothesized that MT1JP may inhibit glioma development by acting as a competing endogenous RNA to modulate the function of miR-24.

To test this hypothesis, a series of experiments were conducted in the present study, and the present results suggested that miR-24 expression was upregulated in glioma tissues and was negatively correlated with MT1JP expression. Additionally, overexpression of MT1JP significantly decreased miR-24 expression. The luciferase reporter assay further confirmed that miR-24 was a direct target of MT1JP. Notably, rescue experiments suggested that the tumor suppressive function of MT1JP in glioma cells may be dependent on miR-24. Xu et al (27) reported that miR-24 promotes glioma cell proliferation by targeting MAX interactor 1, dimerization protein. Collectively, the present results supported this regulatory mechanism, with MT1JP promoting the progression of glioma by acting as a competing endogenous RNA to inhibit the function of miR-24.

In conclusion, the present findings suggested that MT1JP expression was downregulated in glioma tissues compared with that in control normal tissues. Overexpression of MT1JP inhibited glioma cell proliferation and invasion by acting as a competing endogenous RNA and directly sponging miR-24. The present results suggested the important function of MT1JP in glioma development and provided a further understanding of the regulatory mechanisms mediated by competing endogenous RNA underlying glioma development. MT1JP may be 
useful as an important diagnostic biomarker and therapeutic target in glioma.

\section{Acknowledgements}

Not applicable.

\section{Funding}

No funding was received.

\section{Availability of data and materials}

The datasets used and/or analyzed during the present study are available from the corresponding author on request.

\section{Authors' contributions}

JC, JYL and SY performed the majority of the experiments and were the major contributors in writing the manuscript. JC, JL, WL and RZ collected and analysed data. JC, CQ and GD made substantial contributions to the design of the study, drafted the manuscript and revised it critically for important intellectual content. GD gave final approval of the version to be published.

\section{Ethics approval and consent to participate}

The present study was conducted with the approval of the Ethics and Research Committees of The First Affiliated Hospital of Gannan Medical University (Jiangxi, China) and was performed in accordance with the Declaration of Helsinki. All patients provided informed consent.

\section{Patient consent for publication}

Not applicable.

\section{Competing interests}

The authors declare that they have no competing interests.

\section{References}

1. Siegel RL, Miller KD and Jemal A: Cancer statistics, 2018. CA Cancer J Clin 68: 7-30, 2018.

2. Sareddy GR, Viswanadhapalli S, Surapaneni P, Suzuki T, Brenner A and Vadlamudi RK: Novel KDM1A inhibitors induce differentiation and apoptosis of glioma stem cells via unfolded protein response pathway. Oncogene 36: 2423-2434, 2017.

3. Venkatesh HS, Tam LT, Woo PJ, Lennon J, Nagaraja S, Gillespie SM, Ni J, Duveau DY, Morris PJ, et al: Targeting neuronal activity-regulated neuroligin-3 dependency in high-grade glioma. Nature 549: 533-537, 2017.

4. Almiron Bonnin DA, Havrda MC, Lee MC, Liu H, Zhang Z, Nguyen LN, Harrington LX, Hassanpour S, Cheng C and Israel MA: Secretion-mediated STAT3 activation promotes self-renewal of glioma stem-like cells during hypoxia. Oncogene 37: 1107-1118, 2018.

5. Battistelli C, Cicchini C, Santangelo L, Tramontano A, Grassi L, Gonzalez FJ, de Nonno V, Grassi G, Amicone L and Tripodi M: The Snail repressor recruits EZH2 to specific genomic sites through the enrollment of the lncRNA HOTAIR in epithelial-to-mesenchymal transition. Oncogene 36: 942-955, 2017.

6. Zhang Y, Tao Y and Liao Q: Long noncoding RNA: A crosslink in biological regulatory network. Brief Bioinform 2017.
7. Wang X, Vukovic L, Koh HR, Schulten K and Myong S: Dynamic profiling of double-stranded RNA binding proteins. Nucleic Acids Res 43: 7566-7576, 2015.

8. Wang P, Xue Y, Han Y, Lin L, Wu C, Xu S, Jiang Z, Xu J, Liu Q and Cao X: The STAT3-binding long noncoding RNA lnc-DC controls human dendritic cell differentiation. Science 344: 310-313, 2014.

9. Liu L, Yue H, Liu Q, Yuan J, Li J, Wei G, Chen X, Lu Y, Guo M, Luo J and Chen R: LncRNA MT1JP functions as a tumor suppressor by interacting with TIAR to modulate the p53 pathway. Oncotarget 7: 15787-15800, 2016.

10. Xu Y, Zhang G, Zou C, Zhang H, Gong Z, Wang W, Ma G, Jiang $P$ and Zhang W: LncRNA MT1JP suppresses gastric cancer cell proliferation and migration through MT1JP/MiR-214-3p/ RUNX3 axis. Cell Physiol Biochem 46: 2445-2459, 2018.

11. Louis DN, Perry A, Reifenberger G, von Deimling A, Figarella-Branger D, Cavenee WK, Ohgaki H, Wiestler OD, Kleihues P, Ellison DW: The 2016 World Health Organization classification of tumors of the central nervous system: A summary. Acta Neuropathol 131: 803-820.

12. Livak KJ and Schmittgen TD: Analysis of relative gene expression data using real-time quantitative PCR and the 2(-Delta Delta C(T)) method. Methods 25: 402-408, 2001.

13. Boussiotis VA and Charest A: Immunotherapies for malignant glioma. Oncogene 37: 1121-1141, 2018.

14. Stepanenko AA and Kavsan VM: Karyotypically distinct U251, U373, and SNB19 glioma cell lines are of the same origin but have different drug treatment sensitivities. Gene 540: 263-265, 2014.

15. Cao S, Wang Y, Li J, Lv M, Niu H and Tian Y: Tumor-suppressive function of long noncoding RNA MALAT1 in glioma cells by suppressing miR-155 expression and activating FBXW7 function. Am J Cancer Res 6: 2561-2574, 2016.

16. Meng L, Ma P, Cai R, Guan Q, Wang M and Jin B: Long noncodingRNA ZEB1-AS1 promotes the tumorigenesis of glioma cancer cells by modulating the miR-200c/141-ZEB1 axis. Am J Transl Res 10: 3395-3412, 2018.

17. Zong Z, Song Y, Xue Y, Ruan X, Liu X, Yang C, Zheng J, Cao S, Li Z and Liu Y: Knockdown of LncRNA SCAMP1 suppressed malignant biological behaviours of glioma cells via modulating miR-499a-5p/LMX1A/NLRC5 pathway. J Cell Mol Med 8: 5048-5062, 2019.

18. Liu H, Li C, Yang J, Sun Y, Zhang S, Yang J, Yang L, Wang Y and Jiao B: Long noncoding RNA CASC9/miR-519d/STAT3 positive feedback loop facilitate the gliomatumourigenesis. J Cell Mol Med 12: 6338-6344, 2018.

19. Sun Y, Liu J, Chu L, Yang W, Liu H, Li C and Yang J: Long noncoding RNA SNHG12 facilitates the tumorigenesis of glioma through miR-101-3p/FOXP1 axis. Gene: Nov 15, 2018 (Epub ahead of print).

20. Chen X, Li D, Gao Y, Tang W, Iw L, Cao Y and Hao B: Long intergenic noncoding RNA 00152 promotes glioma cell proliferation and invasion by interacting with MiR-16. Cell Physiol Biochem 46: 1055-1064, 2018.

21. Peng WX, Koirala P and Mo YY: LncRNA-mediated regulation of cell signaling in cancer. Oncogene 36: 5661-5667, 2017.

22. Engreitz JM, Haines JE, Perez EM, Munson G, Chen J, Kane M, McDonel PE, Guttman M and Lander ES: Local regulation of gene expression by lncRNA promoters, transcription and splicing. Nature 539: 452-455, 2016.

23. Liu J, Song Z, Feng C, Lu Y, Zhou Y, Lin Y and Dong C: The long non-coding RNA SUMO1P3 facilitates breast cancer progression by negatively regulating miR-320a. Am J Transl Res 9: 5594-5602, 2017.

24. Ding J, YehCR, Sun Y,LinC, Chou J, OuZ, Chang C, Qi J and Yeh S: Estrogen receptor $\beta$ promotes renal cell carcinoma progression via regulating LncRNA HOTAIR-miR-138/200c/204/217 associated CeRNA network. Oncogene 37: 5037-5053, 2018.

25. Tian C, Deng Y, Jin Y, Shi S and Bi H: Long non-coding RNA RNCR3 promotes prostate cancer progression through targeting miR-185-5p. Am J Transl Res 10: 1562-1570, 2018.

26. Sun N, Zhang G and Liu Y: Long non-coding RNA XIST sponges miR-34a to promotes colon cancer progression via Wnt/ $\beta$-catenin signaling pathway. Gene 665: 141-148, 2018.

27. Xu W, Liu M, Peng X, Zhou P, Zhou J, Xu K, Xu H and Jiang S: miR-24-3p and miR-27a-3p promote cell proliferation in glioma cells via cooperative regulation of MXI1. Int J Oncol 42: 757-766, 2013. 